\title{
Educación virtual como herramienta de aprendizaje e innovación en los estudiantes universitarios
}

\section{Virtual education as a tool for learning and innovation in university students}

\author{
Carlos Blanco \\ carloseduardoblanco17@gmail.com \\ https://orcid.org/0000-0003-1783-6069 \\ Edita De Nobrega \\ editasuheil@gmail.com \\ https://orcid.org/0000-0002-6500-7911 \\ Carlos José Blanco \\ Cblanco21@gmail.com \\ https://orcid.org/0000-0002-0332-4698 \\ German Ospino \\ gospino@uc.edu.ve \\ https://orcid.org/0000-0002-5087-6938 \\ Universidad de Carabobo, Valencia - Venezuela
}

Recibido: $26 / 01 / 21$

Aceptado: 28/05/21

\section{Resumen}

Los avances de las tecnologías han permitido la creación de espacios en el proceso de enseñanza aprendizaje. El objetivo del estudio estuvo dirigido a indagar sobre la importancia de la educación virtual como herramienta de aprendizaje e innovación en los estudiantes universitarios. La metodología es documental donde la información fue tomada de varios estudios. Las conclusiones del trabajo han revelado que existen diversas plataformas virtuales en el ecosistema digital, donde los estudiantes realizan sus actividades, mediante la red. La educación virtual constituye una oportunidad para continuar con la formación de los estudiantes en tiempos de complejidad e incertidumbre.

Palabras clave: educación, estudiantes, redes, tecnología, virtualidad.

\section{Abstract}

Advances in technologies have allowed the creation of spaces in the teaching-learning process. The objective of the study was aimed at investigating the importance of virtual education as a tool for learning and innovation in university students. The methodology is 
documentary where the information was taken from various studies. The conclusions of the work have revealed that there are various virtual platforms in the digital ecosystem, where students carry out their activities, through the network. Virtual education is an opportunity to continue with the training of students in times of complexity and uncertainty.

Keywords: Education, Students, Networks, Technology, Virtuality.

\section{Introducción}

El mundo está cambiado y se vive tiempos de incertidumbre. La globalización es una metáfora que expresa la ruptura de lo local, la parcela, de lo regional y lo nacional. Un previo, no se limita a aspectos meramente económico. Se trata, de cualquier manera, de un proceso más complejo y multidimensional.

Es indudable, que los cambios y transformaciones recorren al mundo y se expresan de diversas formas a través de la automatización, el internet, la inteligencia artificial, los teléfonos inteligentes, los vehículos autónomos e híbridos, la nanotecnología, entre otros. Por consiguiente, éstos no son ya una quimera, sino son reales; y con el proceso de globalización en marcha, comienzan a estar a la disposición del planeta.

Por consiguiente, estas tendencias son indetenibles e ilimitadas y por lo tanto, se debe contar con una actitud y una mente abierta con el propósito de comprender que estos cambios vinieron para quedarse y se producirán más temprano que tarde en las universidades, empresas, organizaciones y en la sociedad en general.

En este sentido, el siglo XXI es y será el de la "Sociedad del Conocimiento". Es por ello, desde la década de los ochenta, noventa ha venido surgiendo un nuevo paradigma o un nuevo tipo de sociedad. A ésta se le denomina la Sociedad del Conocimiento. De acuerdo con la Unesco (2005) señala: "La Sociedad del Conocimiento es la sociedad del saber" $(p, 65)$ La sociedad del conocimiento va más allá de la sociedad de la información. De allí, que el conocimiento es un mecanismo que abre el camino hacia la humanización del proceso de mundialización.

Este conocimiento representa la base de desarrollo de las organizaciones de las empresas y de los países en general. Igualmente, Delors (1996) sostiene: "La globalización es el fenómeno más dominante en la sociedad contemporánea y que más influye en la vida diaria de las personas" $(p, 15)$.

De hecho, esto se traduce en el paso de las sociedades industriales a las sociedades del conocimiento donde el eje central lo vienen a constituir la producción del conocimiento y las nuevas tecnologías de la información y la comunicación. Este nuevo tipo de sociedad ha sido estudiada por pensadores como Drucker (1973) Toffler (1960) Nonata y Takeuchi (1980) entre otros. Todos ellos coinciden en que ha surgido un nuevo tipo de sociedad con una nueva economía basada en el conocimiento y la tecnología disruptiva. 
En atención a lo anterior, se viven tiempos de transformaciones. La constante en toda la construcción de cualquier sociedad lo representa es la modificación. Por ello, Heródoto tenia siempre presente ante cualquier circunstancia de la realidad y que lo "único constante es el cambio". De allí, el conocimiento representa un proceso permanente de búsqueda de información. Morín (2000) decía: "El conocimiento en general conlleva el riesgo del error y de la ilusión" $(p, 23)$. Entretanto, el conocimiento hoy en día, representa un proceso de creación, innovación, transformación y crecimiento tanto en el orden personal, como en el orden grupal, así como de la sociedad en su conjunto.

En concordancia con lo anterior, uno de los primeros que acuño el significado de "Sociedad del Conocimiento" fue Drucker (1973) exponía: "Que las nuevas tecnologías de la información y la comunicación acompañan a la Sociedad del Conocimiento y que están transformando radicalmente las economías de mercados, las industrias, los servicios, los puestos de trabajos, y los mercados laborales" $(p, 10)$.

A partir de lo expuesto, la educación virtual, ha sido objeto de muchas acepciones, conceptualizaciones y consideraciones. De acuerdo con autores como Unigarro (2003) Silvio (2004), la denominan como educación en línea, la educación a distancia, la educación virtual, la educación en la red.

Precisamente, Cabero (2006), le da el nombre de educación en la red y otros investigadores, le han acuñado el término de e-learning (Aguilar, 2008). Pero, no ha sido fácil, lograr un consenso dirigido a unificar el nombre de la categoría. No obstante, para el estudio planteado, se conocerá como la educación virtual.

En este contexto, este modelo representa una modalidad que asume la educación y el aprendizaje desde una perspectiva global y holística. Lo anterior, significa que la misma es observada como el resultado de la aplicación del uso de las nuevas tecnologías de la información y comunicación digitales, así como la creación de sistemas de acceso a la red.

Por consiguiente, la educación virtual, representa la acción de los docentes, profesores y estudiantes participan a través de un ecosistema digital de las nuevas tecnologías, plataformas, haciendo uso de las facilitaciones que ofrecen las tecnologías digitales, así como las redes sociales y de tecnológicas de punta.

Ahora bien, que esta nueva forma de desarrollar la educación tiende a modificar los esquemas anteriores de estudio que se venían llevando hasta los actuales momentos. Se está desplazando del aula de clase hacia la educación en línea o en la red; también se incorpora un conjunto de cambios en el aprendizaje al desarrollar el carácter globalizante del proceso educativo en el cual se establece un vínculo con los modelos de simulación y los mecanismos representados por la red.

Precisamente, la educación virtual, por ende, deja a un lado y a su vez rompe con el paradigma de los esquemas de la educación clásica, memorística y tradicional y 
¿eduweb

Revista de Tecnología de Información y Comunicación en Educación • Volumen 15, Nㅜ 2. Mayo-agosto 2021

entonces se genera un nuevo paradigma en el desarrollo del proceso educativo cuyo basamento es más amplio y tiene como punto de apoyo el impulso de las plataformas y redes.

En atención a lo anterior, Cabero (2006) sostiene: "Que la educación basada en las redes una modalidad formativa a distancia que se apoya en la red y además, que facilita la comunicación entre los profesores y los alumnos de acuerdo con determinadas herramientas sincrónicas y asincrónicas de la comunicación" (p.2).

Visto lo anterior, la educación virtual, constituye una modalidad de aprendizaje donde se unen variables como contenidos, las actividades, el nivel educativo, el conocimiento previo de los estudiantes, la comunicación de los participantes con la plataforma tecnológica, así como los distintos dispositivos tecnológicos, los cuales se encuentran conectados a internet.

\section{Diagnóstico}

Para nadie es un secreto, que la pandemia del covid 19, viene afectando al planeta, a los países, así como a las universidades de forma pronunciada, nos agarró con el catarro sin pañuelo, lo que significó que no estábamos preparado para encarar la crisis que género la pandemia. Pero esto, no fue solo al país, sino al mundo.

Ante la realidad expuesta, distintos actores de la comunidad universitaria comparten la idea de que la educación virtual es una herramienta de enseñanza valida en la educación universitaria. Sin menoscabar lo que representa la presencialidad de los docentes en el acompañamiento, en el aprendizaje con los estudiantes. Lo cierto es que algunas universidades pudieron adaptarse, a pesar de los múltiples problemas.

En concordancia con lo anterior, otro de los problemas que ha influido de forma particular para llevar a cabo la enseñanza en los centros de educación superior ha sido la conectividad con el internet. En Venezuela, la verdad es que el país cuenta con un internet bastante débil. Hay poblaciones donde la conectividad es nula. A pesar de los reclamos realizados y hasta ahora los esfuerzos han sido infructuosos.

Por otro lado, migrar de lo presencial a lo digital, tampoco ha sido algo sencillo. Esto requiere de una formación y preparación tanto para los educandos como para los educadores. A ello, súmele, a la carencia de servicios básicos y fallas de energía eléctrica para lograr la conectividad, lo cual se une la debilidad para impulsar la idea de la virtualidad. Carencia de la infraestructura tecnológica, la falta de presupuesto para las universidades, ausencia de equipos e insumos tecnológicos, los bajos salarios de los profesores e investigadores, improvisación, desvalijamientos, hurtos, robos y aunado a lo anterior, se suma los contagios por la pandemia han hecho cuesta arriba llevar a cabo la digitalización. 
Con relación con las universidades privadas; éstas han destinados importantes recursos de sus ingresos y de la matrícula estudiantil para hacer las inversiones necesarias a fin de captar y mantener a sus profesores en el proceso de aprendizaje.

En función de lo anteriormente señalado, esto se encuentra validado en diversos estudios realizados en el 2020, por diversas universidades del país en el cual se han hecho graves inversiones en materia de actualización tecnológica (software, equipos, entre otros) para seguir contribuyendo con el proceso de formación académica universitaria entre los estudiantes universitarios.

En concordancia con lo expuesto, con la educación digitalizada está ampliando la brecha tecnológica entre los estudiantes por la falta de conectividad y la presencia de los equipos tecnológicos que estén disponibles para los educandos y educadores. Tampoco hay subsidios para que los profesores puedan adquirir y contar con equipos actualizados para desarrollar la práctica educativa a distancia.

Por otro lado, no existe voluntad política por parte de los encargados de llevar a cabo la educación digitalizada. Se observa muchas dudas. En algunos espacios se observa, a profesores, maestros aplicando y desarrollando la idea de lo digital, pero de forma aislada, moderada y tímida. Variados pueden ser los motivos, por las cuales la educación digital no es una realidad definitiva en la educación superior.

Entretanto, los argumentos van y vienen, unos a favor y otros en contra. Las cartas se encuentran sobre la mesa y están a la orden de la opinión pública. Sin embargo, hasta ahora, no existe una definición definitiva sobre el destino de la educación virtual en las universidades públicas a raíz de la pandemia. Ojalá que, en los próximos días, se borre los nubarrones sobre el panorama de la digitalización de la educación en las universidades venezolanas.

Por las razones antes señaladas, se producen algunas interrogantes: ¿Cuál es la importancia que genera el desarrollo de la educación virtual en la formación de los estudiantes? ¿Cuáles son los desafíos y retos que producen la educación virtual como herramienta de innovación en los estudiantes en tiempo de complejidad? ¿Por qué la educación virtual se convierte en una alternativa de formación para los estudiantes en tiempos de pandemia en los próximos años?

\section{Objetivos de la Investigación}

\subsection{Objetivo General}

Indagar sobre la importancia de la educación virtual como herramienta de innovación en los estudiantes universitarios, con el fin de mejorar la calidad de la educación en el proceso de enseñanza-aprendizaje. 


\subsection{Objetivos Específicos}

Diagnosticar la situación actual que presenta la educación virtual, para su implementación en el ámbito de la universidad.

Describir las plataformas de educación virtual que utilizan los profesores e investigadores en el ámbito universitario, como consecuencia, de la pandemia que azota al país y al mundo en general.

Promover una cultura virtual para el desarrollo de las nuevas tecnologías de la información y comunicación, así como de las redes sociales como herramientas de aprendizaje de los estudiantes universitarios.

\section{Teoría Sociocultural o Zona del Desarrollo Próximo. Lev Vygotsky.}

Se trata de un enfoque teórico creado por Vygotsky que plantea que la distancia entre el nivel de desarrollo real determinado por la resolución independiente de problemas y el nivel de desarrollo potencial determinado mediante la resolución de problemas bajo la guía de adultos o mediante la colaboración de otros más capaces. De tal forma, que este modelo constituye la más variada gama de habilidades que una persona es capaz de realizar con asistencia, pero aun no puede realizarla de forma independiente. Por ello, es que la interacción con los compañeros juega un rol eficaz en el desarrollo de competencias y estrategias.

Así mismo, los maestros y profesores pueden utilizar ejercicios de aprendizaje conjunto donde los niños menos competentes pueden construir y desarrollar habilidades con la ayuda de sus pares más hábiles, empleando el concepto de zona de desarrollo próximo. Es obvio, entender que otro más informado puede proporcionar una guía crítica y la instrucción necesaria durante el tiempo de aprendizaje sensible. Mientras, un niño que no sea capaz de realizar una tarea por su propia cuenta, si lo seria con la ayuda de una persona o de un instructor calificado.

Por ello, el experto o más informado es con frecuencia el padre, maestro, profesor. No obstante, no siempre tiene que ser así; en múltiples oportunidades los propios pares proveen de la ayuda e instrucción. Inclusive en ciertos períodos de desarrollo del niño, los pares son los referentes más poderosos que los mismos adultos.

Verbigracia, en la adolescencia, una etapa en el cual se forma la identidad y donde los adultos dejan de ejercer influencia sobre los jóvenes y en esta etapa de la vida los jóvenes observan a sus pares para obtener información de cómo actuar, opinar, decidir, modelar, entre otros.

Por último, se aborda la necesidad de la interacción entre compañeros y pares la cual constituye una parte esencial del proceso de aprendizaje para que los niños aprendieran 
nuevas habilidades y con la interacción entre los estudiantes más competentes con personas menos calificadas.

\section{Metodología}

Atendiendo a las características del problema a objeto de estudio y a los objetivos establecidos en el trabajo planteado, debe inscribirse dentro de la investigación de carácter documental y bibliográfica, es decir, que se apoya y se encuentra sustentada en documentos, investigaciones, libros, webside, entre otros.

La delimitación del estudio documental en el marco de la investigación propuesta implica al mismo tiempo que los datos que necesariamente se han de recolectar para alcanzar los objetivos planteados y se obtendrán a partir de la revisión de las fuentes secundarias como son los informes de investigación, documentos relacionados con la problemática planteada.

De la misma manera, Arias (2006) plantea: "La investigación documental es un proceso en la búsqueda, recuperación, análisis, critica, e interpretación, de los datos secundarios, es decir, los obtenidos y registrados por otros investigadores en fuentes documentales, impresas, audiovisuales o electrónica" ( $p, 127)$.

En función de lo anterior, el estudio abordado se dividió en tres (3) fases: en la primera se realizó la revisión bibliográfica que se encuentra vinculada al objeto de estudio; en la segunda fase se analizó los informes y trabajos que están vinculados con la problemática en estudio y la tercera se formularon las aproximaciones, conclusiones y recomendaciones referida a la temática antes señalada.

\section{Resultados}

Los resultados del estudio indican que, al indagar sobre la educación virtual en estos tiempos de incertidumbre, temporalidad y de pandemia del covid 19, constituye herramienta de educación para los estudiantes universitarios, por cuanto permite que los educandos no abandonen, ni se aparten de proceso de aprendizaje. Así mismo, permite desarrollar la interacción y el trabajo colaborativo entre los participantes y, además, que puedan cumplir con las orientaciones emanadas por los organismos internacionales de salud.

Por otra parte, está ampliamente comprobado que los estudiantes de una u otra manera, pueden contribuir y coadyuvar a los profesores con la creación de páginas web, blogs, redes sociales, google meet, marketing digital you tube, zoom, entre otros, donde se aborden los temas relacionados con los contenidos programáticos de los estudios vinculados con el currículo.

Por otro lado, hay una serie de factores que ponen en riesgo el desarrollo de la educación virtual en el ámbito universitario como: la falta de recursos económicos que permitan la

\footnotetext{
28 Educación virtual como herramienta de aprendizaje e innovación en los estudiantes universitarios. -
Eduweb, 2021, mayo-agosto, v.15, n.2./22-31
} 
actualización de la infraestructura tecnológica, falta de energía eléctrica, dificultad de acceso al internet, falta de computadoras, equipos portátiles, los bajos salarios con que cuentan los profesores que les permitan compensar el proceso hiperinflacionario por el cual transita el país, así como la ausencia de un sistema de seguridad social que coadyuve a encarar los complicados problemas que padece y sufre la sociedad venezolana y que ahora se suma la pandemia y el flagelo del coronavirus.

Por las razones anteriores, surge la necesidad de promover en las universidades el uso de una cultura virtual, sin menoscabo la presencia de los profesores en el aula de clase. En este punto, los resultados indican que hay cada vez un consenso generalizado de promover una cultura de innovación permanente. Los estudiantes aprecian y valoran el ejercicio de la educación virtual a tal punto, que se la han exigido a docentes y profesores que ésta sea una herramienta de trabajo orientada a mejorar la calidad de la educación. Todo ello, en sintonía con el acompañamiento y la presencia del profesor en la enseñanza de los educandos.

Justamente, amplios grupos de jóvenes están familiarizados con el uso de los recursos tecnológicos a través de los dispositivos electrónicos como: móviles, celulares, computadores y otros mecanismos digitales dirigidos a cumplir con las tareas sobre el proceso de enseñanza aprendizaje. Por ende, los grandes cambios y transformaciones que se han producido en la humanidad, en materia de creatividad e innovación vienen desde abajo hacia arriba y son el producto de una cultura de emprendimiento con éxitos, fracasos de ensayo y error.

De allí, que en la mayoría de los casos los avances, crecimiento de las naciones, no son producto de la casualidad, ni de un golpe de suerte, ni de improvisaciones, sino que son el resultado de un trabajo constante de dedicación e innovación al estudio que tenga como norte entusiasmar y estimular a las mayorías de estudiantes y jóvenes en la búsqueda de nuevos derroteros de justicia y oportunidades.

\section{Conclusiones}

Después de realizado el estudio, se produjeron algunas aproximaciones y reflexiones derivadas sobre la educación virtual como herramienta de innovación en los estudiantes universitarios que mejore la calidad de la educación en el proceso de aprendizaje. Los resultados antes señalados, indican que deben considerarse como un punto de partida y nunca como algo acabado y definitivo. Que no admite ningún tipo de discusión. Por ello, este estudio representa una contribución que debe ser complementado con el desarrollo de otros y nuevos estudios que sitúen el análisis de la educación virtual en la enseñanza universitaria.

El trabajo demostró que en internet existen diversas plataformas virtuales, las cuales son gratuitas y están a la disposición de los profesores, investigadores y estudiantes universitarios con el propósito de producir una interacción fluida entre los participantes 
del proceso de enseñanza-aprendizaje mediante el mecanismo de intercambio de información, evaluación, retroalimentación de forma sincrónica y asincrónica. Además, es una alternativa válida para encarar y enfrentar el flagelo de la pandemia del covid 19, que sacude al mundo.

Ahora bien, en la investigación, se evidenció que la educación virtual, no es una ciencia ficción, que constituye una realidad, la cual vino para quedarse. Así mismo, es público y notorio que cientos de miles de educandos en el mundo las están utilizando y se capacitan mediante su práctica diaria. De modo, que la educación en línea se ha convertido en una experiencia de trabajo que disminuye costos, acorta distancias y produce resultados.

Por otro lado, nadie pone en duda, que la educación en virtual, todavía presenta y tiene resistencia en algunos sectores que no desean abandonar su comodidad y su zona de confort. No obstante, todo es cuestión de tiempo. El tiempo es el recurso que coloca las cosas en su justa dimensión. Además, que todo tiene su tiempo.

En este contexto, la educación virtual, por tanto, es una herramienta de aprendizaje y formación para los estudiantes. La misma, puede ser utilizada por los maestros, profesores, estudiantes para desarrollar sus actividades mediante el uso del internet, las redes y las plataformas tecnológicas.

Por ello, en el trabajo dado a conocer, se comprobó que, con el uso de la educación en línea y las nuevas tecnologías de la información y comunicación, los estudiantes están proclives a asumir el compromiso ante diversos desafíos planteados con la finalidad de que adquieran aprendizajes necesarios, en la aventura de la experiencia digital.

En atención a lo anterior, el estudio reveló, que a pesar de las aprehensiones que se colocan frente a la educación virtual, algunas de las cuales pueden tener criterios de validez. Sin embargo, esta propuesta de investigación sobre la educación en línea, promueve el desarrollo de una cultura virtual, no solo en los estudiantes, profesores, universidades, sino también en la sociedad en general.

Justamente, en el estudio expuesto, se produjo un hallazgo significativo, donde se demuestra que está ampliando la brecha digital entre los estudiantes, ya que en los actuales momentos carecen de la conectividad de internet, la falta de luz, así como de equipos tecnológicos que permitan impulsar el proceso de formación virtual entre los estudiantes universitarios. Por ello, es necesario tomar en consideración, que esta misma realidad, también la están padeciendo los profesores universitarios.

Desde esta perspectiva, en la investigación se demostró que, ante la complejidad, la temporalidad y la pandemia del covid 19, que recorre al mundo se ha producido un incremento inusitado en el uso de internet, las telecomunicaciones y otros medios tecnológicos cuya alternativa está dirigido a encarar la crisis existente y que no sabemos cuánto tiempo más durará.

\footnotetext{
Educación virtual como herramienta de aprendizaje e innovación en los estudiantes universitarios. -
Eduweb, 2021, mayo-agosto, v.15, n.2./22-31
} 
En conclusión, en cualquier crisis siempre hay oportunidades para impulsar el crecimiento y la formación de los individuos. Hay una sabiduría popular que reza: "Que no hay mal, que por bien no venga". Por lo tanto, uno puede sentirse triste, porque en los rosales, hay espinas o se puede estar alegre porque las rosas que tienen las espinas. Todo va a depender de la actitud o de la manera como se mire las cosas. Ya lo decía Einstein en sus publicaciones. Sin embargo, no es fácil, entender que los obstáculos siempre estarán presentes y que, para llegar a la tierra prometida hay que pasar por el desierto.

\section{Referencias}

Arias, F. (2006). El proyecto de investigación. Guía para su elaboración. Caracas: Editorial Episteme.

Cabero, J. (2006). Bases pedagógicas del e-learning. Revista de Universidad y Sociedad del Conocimiento, http:/scielo.isciii.es/pdf/edu

Cabero, J. (2007). Las nuevas tecnologías en la actividad universitaria. Revista de Medios y Educación. 2381100.

Delors, J. (1996). La educación o la utopía necesaria. La educación encierra un tesoro. Madrid: Ediciones UNESCO.

Drucker, P. (1973). Tecnología, gerencia y sociedad. Petrópolis; vozes

Morín, E. (2000). Los siete saberes necesarios a la educación del futuro. Caracas: Ediciones Faces-UCV.

Unesco. (2005). Educación superior del siglo XXI. Cuba: Ministerio de Educación.

Vygotsky, L. (1979). Desarrollo de procesos psicológicos Superiores. Barcelona 